\title{
Ankle Joint Cooling did not but Knee Joint Cooling altered the Quadriceps and Gastrocnemius Neuromuscular Activation During a Single-leg Drop Jump
}

\author{
Jihong Park ${ }^{1}$ PhD, Kyeongtak Song ${ }^{2}$ PhD, Sae Yong Lee ${ }^{3}$ PhD, Hohyung Ryu ${ }^{4}$ BS \\ ${ }^{1}$ Athletic Training Laboratory, Department of Sports Medicine, Kyung Hee University, Seoul, Korea; ${ }^{2}$ MOTION Science Institute, Department of Exercise and Sport Science, \\ University of North Carolina at Chapel Hill, North Carolina, USA, ${ }^{3}$ Yonsei Institute of Sports Science and Exercise Medicine, Department of Physical Education, Yonsei \\ University, Seoul; ${ }^{4}$ Department of Mathematics, Kyung Hee University, Seoul, Korea
}

PURPOSE:We examined the immediate effect on ankle or knee joint cooling on the lower-extremity activation during a single-leg drop jump.

METHODS: Twenty healthy adults randomly completed three data collection sessions (a 20-minute ankle or knee joint cooling, or control). Two wireless surface electromyography (EMG, 2,000 Hz) electrodes were attached over the vastus medialis (VM) and the medial gastrocnemius (MG) on their right limb. Subjects were asked to drop down from a wooden box (a 30-cm height) on their right foot onto the force platform $(2,000 \mathrm{~Hz})$ and then performed an immediate jump as high as they could. Whole EMG signals (normalised by the mean EMG value recorded during a reference position) within the preactivation (100-ms prior to contact) and the ground contact (on the force platform) during the drop jump task were analysed using functional linear models ( $p=.05$, Cohen's $d$ effect size: ES).

RESULTS: After knee joint cooling, (1) the VM activation was decreased during the ground contact (6 to $14 \%, 30$ to $45 \%, 60$ to $90 \%$ for ES of $0.50,0.47$, and 0.61 , respectively) and (2) the MG activation was increased during the preactivation (84- to 29-ms prior to initial contact, $\mathrm{ES}=1.30$ ) and the ground contact (59 to $100 \%, \mathrm{ES}=1.40$ ). No changes were observed under the condition of ankle joint cooling or control.

CONCLUSIONS: A 20-minute unilateral knee joint cooling may result in an ipsilateral quadriceps inhibition during a drop jump task. The gastrocnemius appears to increase activation as a compensatory strategy responding to the quadriceps inhibition. The altered neuromuscular activations do not seem to put an individual at risk of injury, resulting from compressive forces in the sagittal plane.

Key words: Electromyography, Preactivation, Drop landing, Functional data analysis

\section{INTRODUCTION}

Cold application, also known as cryotherapy, is an effective modality to reduce metabolic rate and pain after orthopaedic injury [1]. Applying cooling agents also allows patients to perform pain-free stretch or exercise, so-called cryo-stretch [2] or -kinetics [3]. Additionally, an increase of muscle function is another clinical advantage in using cold application that an increase in quadriceps function after knee joint cooling was reported in patients with knee osteoarthritis [4]. Similarly, this facilitatory (increased above the baseline) effect was also observed in healthy individuals that ankle or knee joint cooling resulted in an increase of the solues [5] or quadriceps activation [6,7].

Based on the aforementioned facilitatory effect [6,7], joint cooling-induced performance enhancement in 20-m sprint [8] or maximal vertical jump [9] have been examined. Particularly, healthy subjects ran 0.09-second faster in 20-m sprint after a 20-minute of bilateral knee joint cool-

Corresponding author: Jihong Park Tel +82 312012721 Fax +82 312048117 E-mail jihong.parkakhu.ac.kr

*This work was supported by the National Research Foundation of Korea Grant funded by the Korean Government (NRF-2014S1A5A8019804).

Keywords Electromyography, Preactivation, Drop landing, Functional data analysis

Received 16 Jun 2020 Revised 8 Jul 2020 Accepted 12 Jul 2020

(a) This is an Open Access article distributed under the terms of the Creative Commons Attribution Non-Commercial License (https://creativecommons.org/licenses/by-nc/4.0/) which permits unrestricted non-commercial use distribution, and reproduction in any medium, provided the original work is properly cited. 
ing [8]. After a temperature reduction due to cooling, an increase in thermoreceptor activity (e.g., Krause's end bulb) initiates change in afferent sensory input [10] to the central nervous system. At the spinal level, altered sensory information interacts with the interneurons (e.g., Ia and Ib inhibitory or excitatory interneurons: also receive from other afferent/ efferent signals and the other interneurons) [11], which in turn increase facilitatory (excitatory) response of the motoneuron pool as the net effect [7]. In this neurological process, decreased recruitment threshold of inhibited motoneurons [5] and masking inhibitory synaptic signals [4] have been suggested as the mechanisms behind the net effect in excitatory descending signals.

While the use of lower-extremity joint cooling is getting popular for either purpose of a therapeutic effect or performance enhancement, any potential harmful effect (e.g., increasing risk of injury) due to the neuromuscular alterations needs to be examined. Further, current literature does not provide information as how lower-extremity muscles neuromuscularly respond to ankle or knee joint cooling. Hence, in the primary purpose of this study was to observe how a 20-minute unilateral ankle or knee joint cooling alters neuromuscular activity in the quadriceps and gastrocnemius during a single-leg drop jump contact (from an initial ground contact to jump-off) and preactivation (100-ms prior to the initial contact), as compared to a non-cooling condition (control). Secondarily, we examined if altered neuromuscular patterns in response to either joint cooling during the drop jump between females and males were different. Understanding the influence of the sex difference would help establish prevention or training strategies. We had our subjects perform a single leg-drop jump because it is a functional plyometric movement consisting of the eccentric and concentric contraction phases. Based on the previous reports $[5,6]$, we hypothesised that ankle or knee joint cooling would result in a facilitation of the gastrocnemius or quadriceps, respectively during the preactivation and ground contact.

\section{METHODS}

\section{Subjects}

Twenty healthy physically active adults (10 females and 10 males; age: $22.8 \pm 3.0$ years, height: $169.0 \pm 8.6 \mathrm{~cm}$, mass: $65.9 \pm 12.2 \mathrm{~kg}$ ) participated in this crossover study. Prior to participation, all subjects signed an informed consent, approved by the university's institutional review board. According to the inclusion criteria, all subjects had to be free of any history of lower-extremity injury for the past six months and orthopaedic surgery for the lifetime. Exclusion criteria included any neuromuscular disorder or cardiopulmonary disease.

\section{Procedures}

After reading the testing procedures of the study, providing informed consent, and changing clothes (standardised spandex shorts and shirts, tennis shoes, and socks), subjects performed several practice trials of a single-leg drop jump task. The single-leg drop jump was defined as a drop down from a $30-\mathrm{cm}$ height wooden box $(55 \times 55 \times 30 \mathrm{~cm})$ on their right foot onto the floor-embedded force platform followed by an immediate vertical jump as high as possible, and then landing back on the same force platform. After skin site was shaved and cleaned, two wireless EMG electrodes (Trigno Wireless Systems, Delsys Inc., Natick, MA USA) were attached over the right-leg muscle bellies of the vastus medialis (VM) and the medial gastrocnemius (MG) in a previously recommended manner [12]. With each testing session, subjects were then asked to squat down until they barely touch their butt on the edge of table (height of $0.7 \mathrm{~m}$ ) and maintained this position for four seconds [13]. This semi-squat activity was performed for normalisation of EMG amplitude that the EMG amplitude during the drop jump task was divided by the EMG amplitude during the isometric reference position. Three successful trials of the single-leg drop jump tasks were recorded at pre- and post-measurements. After pre-measurements, one of the three conditions (a 20-minute ankle or knee joint cooling in the right limb, or control-no cooling) were administered, in a counterbalanced order. For joint cooling, two plastic bags $(20.3 \times 40.6 \mathrm{~cm}$ : filled with $1 \mathrm{~L}$ crushed ice $)$ were directly secured by an elastic wrap (Fig. 1). The plastic bags for the ankle joint were placed on the medial and lateral aspects while knee joint were on the anterior and posterior aspects [8]. For the control condition, subjects remained in the seated position for 20-minute without cooling.

\section{Data collection and reduction}

The ground contact (from initial contact to jump off) was determined



Fig. 1. Ankle or knee joint cooling. 
by the vertical GRF (exceeded $10 \mathrm{~N}$ ) from the force platform (AMTI, Watertown, MA, USA; sampled at 2,000 Hz, synchronised with the EMG signals). Within the data of the ground contact, landing and jumping phases were determined by the peak knee flexion in the sagittal plane (calculated by joint kinematics from 3D motion analysis, which is not reported in this study). For the preactivation, the data of 100 -ms period prior to initial contact were selected. All EMG analogue signals were band-pass filtered $(20-450 \mathrm{~Hz}$ ) with a gain of 1,000 (rejection ratio: $>80 \mathrm{~dB}$ ) and digitally sampled at a frequency of 2,000 Hz. Recorded raw EMG data were further smoothed using a root mean square algorithm with a 15 -ms moving window. Smoothed EMG amplitude within the preactivation and the ground contact phases during the drop jump was normalised by the mean of smoothed EMG value during the isometric reference position sampled at each session.

\section{Statistical analysis}

To test joint cooling effect over time on the VM and MG neuromuscular activation during the preactivation and the ground contact, we performed functional analysis of variance [14] using time-registration polynomial function (curve). To determine statistical significances, we then plotted the mean difference and the corresponding estimation of $95 \%$ confidence bands in each pairwise comparison $(p<.05)$. Statistical differences were considered if the 95\% confidence bands did not cross zero. The corresponding mean difference indicated the magnitude of difference. Since this statistical approach comprehensively evaluates the whole data as a function, it is considered as a superior over traditional methods by analysing discrete values peaked or averaged during a certain time period. Dependent variables showing statistical differences were analysed (the same functional data analysis) again using the separate data set of females and males. This secondary analysis allowed us to determine whether sex-differences influenced the results. To determine practical significance, Cohen's d effect sizes (ES) were calculated for the time period with all detected statistical significances [15]. Statistical package R (version 3.4.3, R development core team) was used for all statistical analyses.

\section{RESULTS}

The average EMG amplitude recorded during the 4-second isometric reference position for each session are shown in Fig. 2A. We did not observe any neuromuscular change under the condition of the ankle joint cooling or control. After knee joint cooling, we observed decreased neuromuscular activity in the VM during landing (an average reference value of $0.8 \%$ during 6 to $14 \%$, an average ES of 0.50 ; an average reference value of $1.3 \%$ during 30 to $45 \%$, an average ES of 0.47 ) and jumping (an average reference value of $1.8 \%$ during 60 to $90 \%$, an average ES of 0.61 ) phase (Fig. 2B). We also observed an increase in the MG preactivation (84 to 29-ms prior to the initial contact: an average reference value of 6.5\%, an average ES of 1.30: Fig. 2C) and during the jumping (an average reference value of $10.6 \%$, during 56 to $100 \%$, an average ES of 1.30: Fig. 2D) phase.

Specifically, females had an increase in the VM activation for $65 \%$ of the time during ground contact (an average reference value of $2.4 \%$ with an average ES of 0.69: Fig. 3B) while males only showed a corresponding value of $23 \%$ (an average reference value of $1.6 \%$ with an average ES of 0.48: Fig. 3C). In regard to the MG activation, females showed greater magnitude of neuromuscular alterations during preactivation (an average reference value of $1.7 \%, 45 \%$ of the time, with an average ES of 0.38 : Fig. 3E) and ground contact (an average reference value of 14.4\%, during ground contact of 59-100\%, with an average ES of 0.43: Fig. $3 \mathrm{H}$ ) than males did during preactivation (an average reference value of $5.9 \%, 6 \%$ of time, with an average ES of 0.33: Fig. 3F) and ground contact (an average reference value of $12.0 \%$, during $79-86 \%$, with an average ES of 0.36: Fig. $3 \mathrm{I})$.

\section{DISCUSSION}

Our hypothesis that the VM or MG activation would increase after a 20-minute unilateral ankle or knee joint cooling, respectively, during a single-leg drop jump task was not supported. The conditions with ankle joint and non-cooling (control) did not alter neuromuscular activity in the VM and MG. Our study was the first attempt to directly compare change in neuromuscular activation after ankle or knee joint cooling during a drop jump task, to a condition of no joint cooling (controls). Contrary to what we expected, subjects' VM activation was decreased after knee joint cooling. The direction of altered VM activation is not consistent with previous reports $[16,17]$ that there was no activation change after knee joint cooling. Altered afferent sensory input along with an increased activation of cold-sensitive thermoreceptors (e.g., Krause's end bulbs) [10] are thought to cause neuromuscular alterations in our study. Once the altered sensory afferent nerve fibres arrived to the spinal cord, neural interactions with the interneurons (connected with $a$ 
EMG amplitude during the reference position
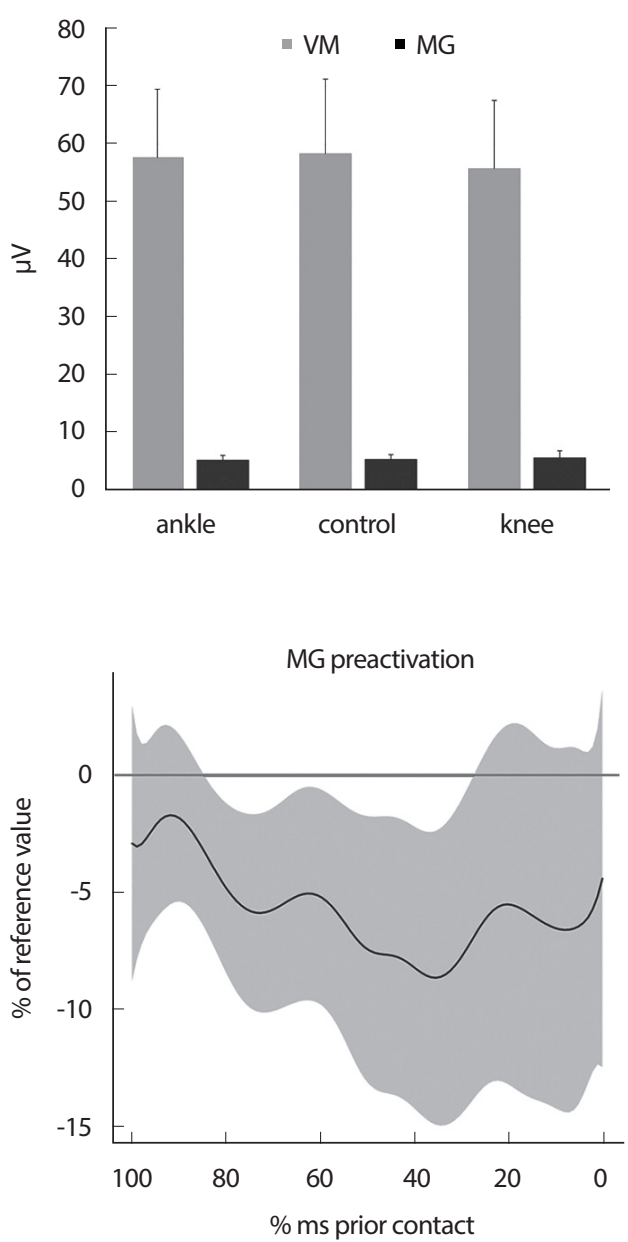
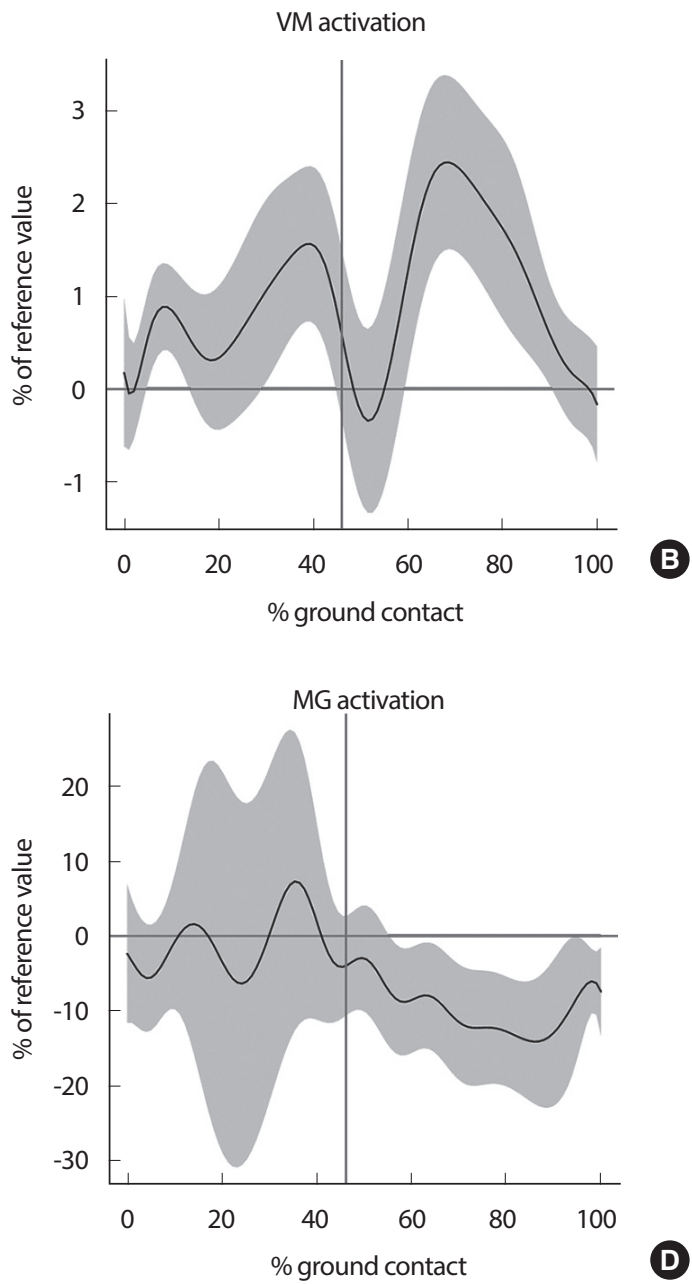

Fig. 2. Changes in neuromuscular activities in the VM and MG after knee joint cooling. (A) The EMG amplitudes during the reference position. Error bars indicate $95 \%$ confidence intervals. (B) The VM activation was decreased during the landing (an average reference value of $0.8 \%$ and $1.3 \%$, with an average ES of 0.50 and 0.47 , during 6 to $14 \%$ and 30 to $45 \%$ of ground contact, respectively) and jumping (an average of $1.8 \%$, with an ES of 0.61 , during 60 to $90 \%$ of ground contact) phase. (C) The MG activation was increased 84- to 29-ms prior to the initial contact (an average reference value of $6.5 \%$, with an ES of 1.30). (D) The MG activation was increased (an average reference value of $10.6 \%$, with an ES of 1.40 ) during the jumping phase (56 to $100 \%$ of ground contact).

-and $\gamma$-motor neurons) may have led to the VM inhibition and MG facilitation [11] in our study. In this spinally mediated process, although speculated, a regulation of the amount of Ib inhibitory signals [18] and/ or $\gamma^{-}$[7] loop [19] are thought to be the contributing factors. While aforementioned neurophysiological effects of joint cooling were similar to those in previous studies [16,17], disagreement in the results could be explained by the different methodologies of data reduction and statistical analysis. While the previous results were from the traditional statistical analysis using discrete values representing the specific time intervals while we performed a functional data analysis using the entire data within the time period.

Changed neuromuscular activations in the VM and MG could have been affected by sex-specific recruitment patterns due to joint cooling.
The results showed that females were affected more than males (Fig. 3). Given that there were at least 11 subjects in the previous studies concerning sex-differences on muscle activations [20-22], we suggest that our results (10F; 10M) are statistical trends. While the direction of neuromuscular alterations was not different (a decrease in VM; an increase in MG), the combined results (sex-collapsed: Fig. 3A, D, G) were quit similar to those in females (Fig. 3B, E, H). This suggests that the magnitude of altered VM and MG neuromuscular activity due to a joint temperature reduction may be greater in females than in males. While not directly measured, we believe that the greater neuromuscular changes in females were from the amount of quadriceps and gastrocnemius (e.g., cross-sectional area), and the previous experience on activities similar to the drop jump task and cryotherapy. Future study should attempt if this 



VM activation during ground contact
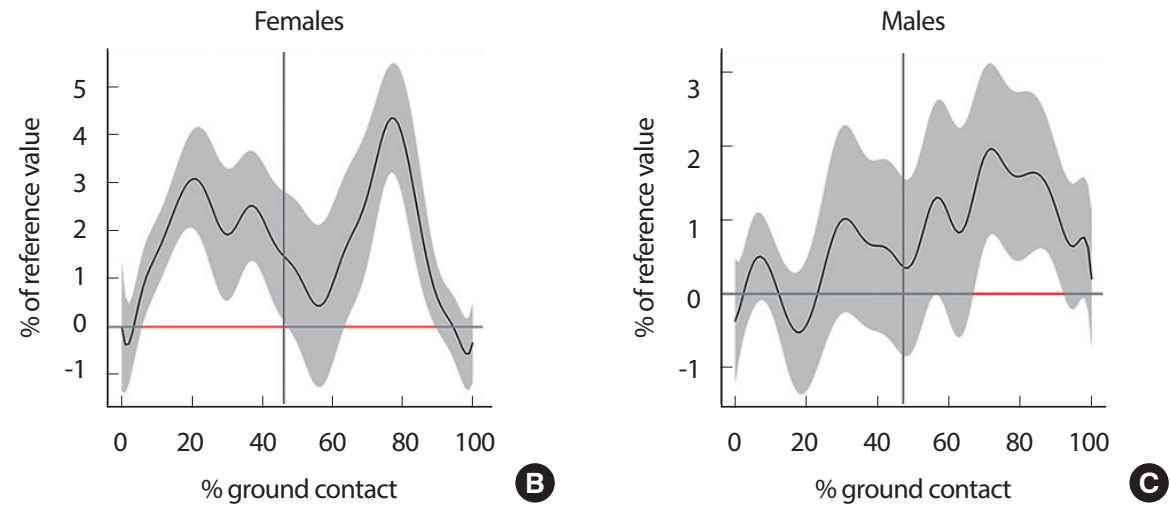

MG activation during preactivation

Females
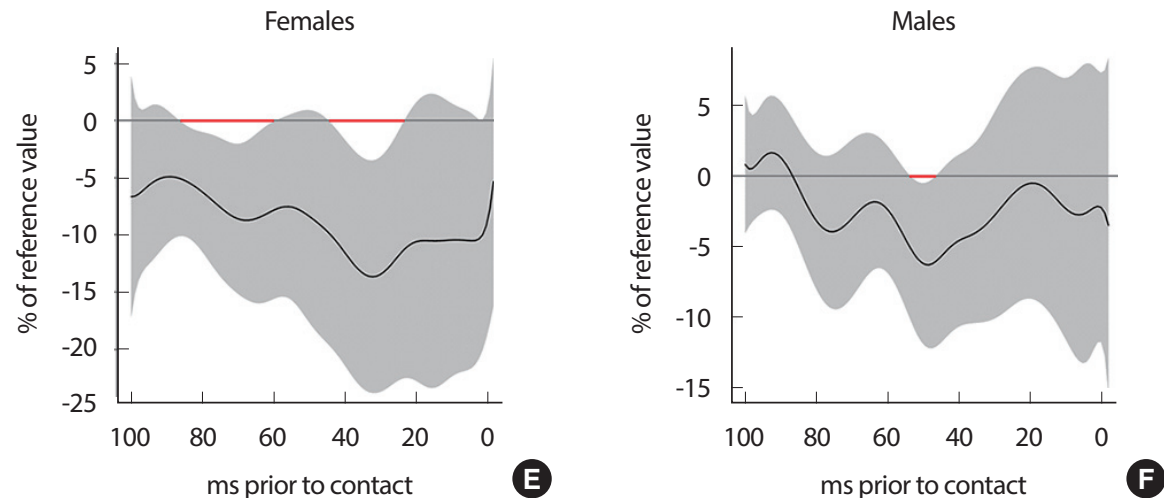

MG activation during ground contact

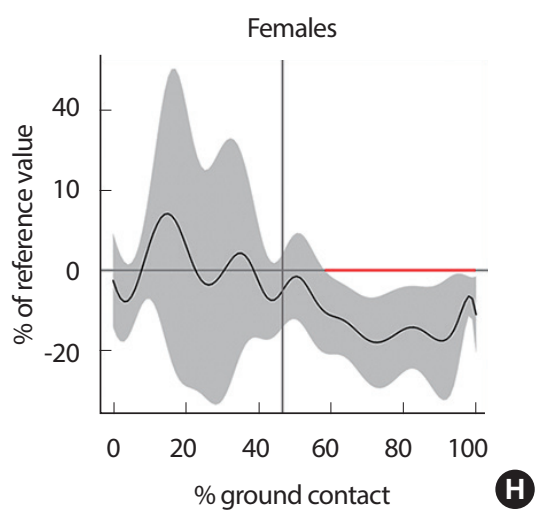

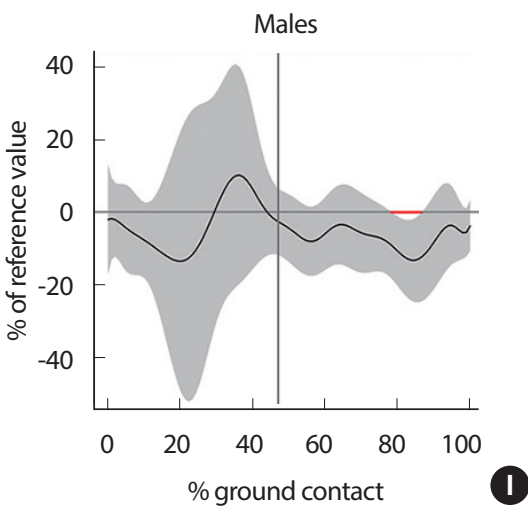

Fig. 3. Sex differences in trials that showed statistical significances. The Figs. in the left column (A, D, and G: See Fig. 1 for detailed information in neuromuscular changes) are the combined results $(n=20)$. On the same row, the Figs. at the middle and right indicate the results of separate analysis for females $(n=10)$ and males $(n=10)$, respectively. (B) The VM activation in females was decreased during the ground contact (an average reference value of $2.2 \%$, with an average ES of 0.66 , during $7-47 \%$ of landing; an average reference value of $2.8 \%$, with an average ES of 0.72 , during $64-86 \%$ of jumping phase). (C) The VM activation in males was decreased during the jumping phase (an average reference value of $1.6 \%$, with an average ES of 0.48 , during $68-91 \%$ of ground contact). (E) The MG activation in females was increased 86- to 61-ms and 46 to $26 \mathrm{~ms}$ prior to the initial contact, respectively (an average reference value of $1.2 \%$ and $2.1 \%$, with an ES of 0.38 for both, respectively). (F) The MG activation in males was increased 55- to 49-ms prior to the initial contact (an average reference value of $5.9 \%$ and $2.1 \%$, with an ES of 0.33 ). (H) The MG activation in females was increased during ground contact (an average reference value of $14.4 \%$, with an ES of 0.43 , during $59-100 \%$ of jumping phase). (I) The MG activation in males was increased during ground contact (an average reference value of $12.0 \%$, with an ES of 0.36 , during $79-86 \%$ of jumping phase). 
difference in muscle recruitment patterns is affected by muscle mass, training level, or familiarity with a movement task.

Ankle joint cooling did not change any neuromuscular activation. Previous ankle joint cooling resulted in a plantarflexor facilitation - an increase in the soleus H-reflex [23] and plantarflexion torque [5]. Based on this evidence, we thought that there would be a similar change in the MG neuromuscular activation and expected to see how the quadriceps would respond to it. However, our participants maintained the normal muscle activation in their VM and MG. These results could be explained by the functional movement task that our participants performed. It was a high velocity multi-joint weight-bearing activity that the average peak vertical GRF was 3.9 times that of body weight in our participants. A possibly produced facilitatory effect (e.g., an increase in plantarflexion torque) that was insufficient to contribute to any change in neuromuscular activity [9]. Additionally, cold-induced sensitive afferents (thought to trigger a plantarflexor facilitation) could be masked by other sensory inputs relating to postural control and movements during the drop jump [23].

Knee joint cooling altered neuromuscular activations that the VM was inhibited (ES: 0.32 to 0.83 ) whereas the MG was facilitated (both preactivation and ground contact; ES: 1.0 to 1.8). A neuromuscular relationship between the quadriceps and triceps surae has been reported $[24,25]$. While a quadriceps inhibition soon after a knee joint injury is common [26-28], an increased soleus activation is thought to compensate for weakened lower-extremity muscle function [28]. Based on previous reports [6,7], we expected an increase in quadriceps activation but it actually showed a reduced activation. Interestingly, our participants showed VM inhibition in both landing (6 to 14\%; 30 to $45 \%$ of ground contact) and jumping (60 to $90 \%$ of ground contact) phases. Therefore, the results of previous studies $[6,8,23]$ and the current study may suggest that neuromuscular alterations after knee joint cooling are determined by complex factors such as weight-bearing, multi-joint action, joint angular velocity, and co-contractions. The VM inhibition due to knee joint cooling, and the relationship between VM and MG during other functional movements should be examined in the future. The MG facilitation was observed during preactivation (84 to 29 -ms prior to initial contact) and ground contact (56 to $100 \%$ ). We believe that this is a compensatory response to the VM inhibition that allows maintenance of an upright posture and completion of the jump task [28]. Preactivation is considered as a preparatory neuromuscular process, which is also known as feed forward system [20]. The amount of MG preactivation has a linear relationship with the vertical displacement during a drop land task [29] and an inverse relationship with fatigue [21]. Additionally, the preactivation in plantarflexors plays an antagonist role in ACL (anterior cruciate ligament) injury [30,31]. Therefore, our results (an increased MG preactivation in responding to $\mathrm{VM}$ inhibition) can be interpreted as a protective mechanism.

A joint compressive force alone, without valgus or rotational force, can result in an ACL injury [32]. In this situation, quadriceps force can attenuate the axial compressive force threshold for an ACL rupture [33] With that being said, the observed VM inhibition after knee joint cooling in our subjects may imply that the opposite direction of injury prevention. The amount of VM EMG amplitude recorded during the isometric reference position (Fig. 2A) was equivalent to a $20 \%$ of maximal voluntary contraction during a seated knee extension or leg press exercise [34]. The MG amplitude was approximately ten times less than VM (57.3 vs. $5.5 \mu \mathrm{V}$ : Fig. 2A). Isolated compressive forces, resulting in ACL rupture or tibial fracture, were previously reported as a range between 9,743-11,604 N [33]. While the average peak vertical GRF during the single-leg drop jump in our subjects were approximately $2,500 \mathrm{~N}$, a $20 \%$ reduction in quadriceps force may indicate that the compressive force applied to the knee joint would go up to 3,000 N (assuming that 2,500 N was recorded by performing a drop jump task with maximum quadriceps strength). Although not directly converted, the total amount of compressive force after knee joint cooling during the land jump task in our study $(3,000 \mathrm{~N})$ is much less than the 9,743 $\mathrm{N}$ [33]. Therefore, observed neuromuscular alterations (no greater than 2 and $11 \%$ of the reference value in the VM and MG activations, respectively) are unlikely to lead to an injury although calculated effects sizes wee moderate to strong. An important limitation should be noted that we only observed change in neuromuscular activation in the sagittal plane. Our conclusion that the probability of injury due to compressive force alone is less likely based on an assumption that other forces were acting on the joint. While a dynamic valgus [35] and rotational forces [36] also contribute an ACL injury, more data (e.g., muscles acting on the frontal plane) would be necessary to expand the results.

\section{CONCLUSIONS}

While ankle joint cooling did not show any change in neuromuscular activation, a 20-minute unilateral knee joint cooling resulted in a VM inhibition (during ground contact) and MG facilitation (during preacti- 
vation and ground contact) during a single-leg drop jump task. Females appear to have greater magnitude of neuromuscular alterations due to knee joint cooling than males. The altered neuromuscular activations do not seem to put an individual at risk of injury, resulting from compressive forces in the sagittal plane.

\section{CONFLICT OF INTEREST}

The authors declare that they do not have conflict of interest.

\section{AUTHOR CONTRIBUTIONS}

Conceptualization: J Park; Data curation: K Song; Methodology: J Park, K Song, SY Lee, H Ryu; Visualization: J Park, H Ryu; Writingoriginal draft: J Park; Writing - review \& editing: J Park, K Song, SY Lee, H Ryu.

\section{ORCID}

$\begin{array}{ll}\text { Jihong Park } & \text { https:/orcid.org/0000-0002-8844-3855 } \\ \text { Kyeongtak } & \text { https://orcid.org/Song 0000-0002-0671-2127 } \\ \text { Sae Yong } & \text { https://orcid.org/Lee 0000-0002-0526-3243 } \\ \text { Hohyung Ryu } & \text { https://orcid.org/0000-0003-4735-4861 }\end{array}$

\section{REFERENCES}

1. Bleakley CM, Costello JT. Do thermal agents affect range of movement and mechanical properties in soft tissues? A systematic review. Arch Phys Med Rehabil. 2013;94(1):149-63.

2. Sefiddashti L, Ghotbi N, Salavati M, Farhadi A, Mazaheri M. The effects of cryotherapy versus crystretching on clinical and functional outcomes in athletes with acute hamstring strain. J Bodyw Mov Ther. 2018;22(3):805-9.

3. Kaur B, Kaushal K, Kaur S. Effect of cryokinetics on talofibular ligament of improving proprioception of the ankle joint among sports person having ankle sprain. Indian J Physiother Occup Ther. 2019;13(2): $180-5$.

4. Pietrosimone BG, Hart JM, Saliba SA, Hertel J, Ingersoll CD. Immediate effects of transcutaneous electrical nerve stimulation and focal knee joint cooling on quadriceps activation. Med Sci Sports Exerc. 2009;41(6):1175-81.
5. Hopkins JT, Stencil R. Ankle cryotherapy facilitates soleus function. J Orthop Sports Phys Ther. 2002;32(12):622-7.

6. Pietrosimone BG, Ingersoll CD. Focal knee joint cooling increases the quadriceps central activation ratio. J Sports Sci. 2009;27(8):873-9.

7. Rice D, McNair PJ, Dalbeth N. Effects of cryotherapy on arthrogenic muscle inhibition using an experimental model of knee swelling. Arthritis Rheum. 2009;61(1):78-83.

8. Kim H, Lee D, Choi HM, Park J. Joint cooling does not hinder athletic performance during high-intensity intermittent exercise. Int J Sports Med. 2016;37(8):641-6.

9. Lee M, Kim S, Choi HM, Park J. Ankle or knee joint cooling alters countermovement but not squat jump height in healthy collegiate athletes. Isokinet Exerc Sci. 2017;25(1):1-8.

10. Nadler SF, Weingand K, Kruse RJ. Ther physiologic basis and clinical applications of cryotherapy and thermotherapy for the pain practitioner. Pain Physician. 2004;7(3):395-9.

11. Hopkins JT, Ingersoll CD. Arthrogenic muscle inhibition: a limiting factor in joint rehabilitation. J Sport Rehabil. 2000;9(2):135-59.

12. Hermens HJ, Disselhorst-Klug C, Rau G. Development of recommendations for SEMG sensors and sensor placement procedures. J Electromyogr Kinesiol. 2000;10(5):361-74.

13. Moss WR, Feland JB, Hunter I, Hopkins JT. Static stretching does not alter pre and post-landing muscle activation. Sports Med Arthrosc Rehabil Ther Technol. 2011;3(1):9.

14. Park J, Seeley MK, Francom D, Reese CS, Hopkins JT. Functional vs. traditional analysis in biomechanical gait data: an alternative statistical approach. J Human Kinet. 2017;60(1):39-49.

15. Cohen J. Quantitative methods in psychology. Psychol Bull. 1992;112 (1):155-9.

16. Hart JM, Leonard JL, Ingersoll CD. Single-leg landing strategy after knee-joint cryotherapy. J Sport Rehabil. 2005;14(4):313-20.

17. Schmid S, Moffat M, Gutierrez GM. Effect of knee joint cooling on the electromyographic activity of lower extremity muscles during a plyometric exercise. J Electromyogr Kinesiol. 2010;20(6):1075-81.

18. Hopkins JT, Ingersoll CD, Edwards JE, Klootwyk TE. Cryotherapy and transcutaneous electric neuromuscular stimulation decrease arthrogenic muscle inhibition of the vastus medialis after knee joint effusion. J Athl Train. 2001;37(1):25-31.

19. Rice DA, McNair PJ. Quadriceps arthrogenic muscle inhibition: neural mechanisms and treatment perspectives. Semin Arthritis Rheum. 2010;40(3):250-66. 
20. DeMont RG, Lephart SM. Effect of sex on preactivation of the gastrocnemius and hamstring muscles. Brit J Sports Med. 2004;38(2):1204.

21. Gehring D, Melnyk M, Gollhofer A. Gender and fatigue have influence on knee joint control strategies during landing. Clin Biomech. 2009;24(1):82-7.

22. Krishnan C, Williams GN. Sex differences in quadriceps and hamstrings EMG-moment relationships. Med Sci Sports Exerc. 2009;41 (8):1652-60

23. Kim K-M, Ingersoll CD, Hertel J. Facilittaion of Hoffmann reflex of ankle muscles in prone but not standing positions by focal ankle-joint cooling. J Sport Rehabil. 2015;24(2):130-9.

24. Hopkins JT, Ingersoll CD, Edwards JE, Cordova ML. Changes in soleus motoneuron pool excitability after artificial knee joint effusion. Arc Phys Med Rehabil. 2000;81(9):1199-203.

25. Koceja DM. Quadriceps mediated changes in soleus motoneuron excitability. Electromyogr Clin Neurophysiol. 1995;35(1):25-30.

26. Palmieri-Smith RM, Kreinbrink J, Ashton-Miller JA, Wojtys EM. Quadriceps inhibition induced by an experimental knee joint effusion affects knee joint mechanics during a single-leg drop landing. Am J Sports Med. 2007;35(8):1269-75.

27. Park J, Hopkins JT. Induced anterior knee pain immediately reduces involuntary and voluntary quadriceps activation. Clin J Sport Med. 2013;23(1):19-24.

28. Hopkins JT, Ingersoll CD, Krause BA, Edwards JE, Cordova ML. Effect of knee joitn effusion on quadriceps and soleus motoneuron pool excitability. Med Sci Sports Exerc. 2001;33(1):123-6.
29. Mrdakovic V, Ilic DB, Jankovic N, Rajkovic Z, Stefanovic D. Pre-activity modulation of lower extremity muscles within different types and heights of deep jump. J Sports Sci Med. 2008;7(2):269-78.

30. Adouni M, Shirazi-Adl A, Marouane H. Role of gastrocnemius activation in knee joint biomechanics: gastrocnemius acts as an ACL antagonist. Comput Methods Biomech Biomed Engin. 2016;19(4):376-85.

31. Elias JJ, Faust AF, Chu Y-H, Chao EY. The soleus muscle acts as an agonist for the anterior cruciate ligament. Am J Sports Med. 2003;31 (2):241-6.

32. Meyer EG, Gaumer TG, Slade JM, Smith JC, Haut RC. Tibiofemoral contact pressures and osteochondral microtrauma during anterior cruciate ligament rupture due to excessive compressive loading and internal torque of the human knee. Am J Sports Med. 2008;36(10): 1966-77.

33. Wall SJ, Rose DM, Sutter G, Belkoff SM, Boden BP. The role of axial compressive and quadriceps forces in noncontact anterior cruciate ligament injury. Am J Sports Med. 2012;40(3):568-73.

34. Alkner BA, Tesch PA, Berg HE. Quadriceps EMG/force relationship in knee extension and leg press. Med Sci Sports Exerc. 2000;32(2):45963.

35. Shin CS, Chadhari AM, Andriacchi TP. Valgus plus internal rotation moments increase anterior cruciate ligament strain more than either alone. Med Sci Sports Exerc. 2011;43(8):1484-91.

36. Hewett TE, Myer GD, Ford KR, Heidt Jr RS, Colosimo AJ, et al. Biomechanical measures of neuromuscular control and valgus loading of the knee predict ACL injury risk in female athletes. Am J Sports Med. 2005;33(4):492-501. 